Journal of Mathematics and Informatics

Vol. 18, 2020, 51-63

ISSN: 2349-0632 (P), 2349-0640 (online)

Published 28 January 2020

Journal of

www. researchmathsci.org

DOI: http://dx.doi.org/10.22457/jmi.v18a5162

Mathematics and

Informatics

\title{
Modelling Predator Prey with Diffusion for Migrating Wildebeest and Lion in the Presence of Drought
}

\author{
Kisoma Linus, N." and Kuznetsov Dmitry \\ School of Computational and Communication Science and Engineering \\ Nelson Mandela African Institution of Science and Technology \\ P.O.Box 447, Arusha Tanzania \\ Corresponding author. Email: linuskisoma@gmail.com
}

Received 12 December 2019; accepted 14 January 2020

\begin{abstract}
In this paper we present a two species prey predator reaction-diffusion model with Holling II functional response to study the effects of drought to the dynamical behaviour of wildebeest (Connochaetustaurinus) and lion (pantheraleo) in the Serengeti ecosystem. We discretized our model equations by the implicit Euler method and the numerical simulations were performed in Matlab. The major limiting factor for wildebeest abundance is availability of enough forage and predation. We varied the parameter of drought for prey and predator and observed that drought reduces the abundance of both species. If drought persists then it is likely to lead to extinction of both species. Further we found spatial driven variabilities arising when both wildebeest and lion move through diffusion processes. The results predict that diffusion of the wildebeest is motivated by search for better forage and avoiding predators while the diffusion of lion is motivated by hunting the prey. Our model agrees with different theoretical and empirical studies about the environmental effects to the survival of wildebeests and lions.
\end{abstract}

Keywords: migration, diffusion, drought

AMS Mathematics Subject Classification (2010): 92D40

\section{Introduction}

The great migration of wildebeest (Connochaetestaurinus) from Serengeti national park in northern Tanzania to Masai-Mara in Kenya followed by other ungulates and predators is so distinctive that it has attracted researchers and visitors around the world [1]. The great migration is an annual movement of millions of animals searching for better forage $[1,2]$. Prey-predator mathematical models have been extensively studied by many authors [3]. Ecological studies in the Serengeti ecosystem have focused mainly on factors such as poaching, retaliatory killings, prey refuge diseases outbreak such as the study of $[1,3,4,5]$. Only a few studies included mathematical models such as the study of [1]. These studies relied mainly on explaining some factors such as food, predation and sustainable harvesting strategies of wildebeest and other herbivores species in the Serengeti ecosystem. None of these studies included the aspect of diffusion (migration) in the mathematical model. Migration of wildebeest and other species touches different patches. We included diffusion in the model to capture dynamics such as the existence of a 


\section{Kisoma Linus, N. and Kuznetsov Dmitry}

minimal patch size necessary to support a population, the presence of traveling wave fronts corresponding to biological invasions, and the formation of spatial patterns[7].

Drought has been reported to limit wildebeest abundance. Since 1960's wildebeest population has been fluctuating to about 1.3 million until the year 1993 when a severe drought reduced the wildebeest population to about 917,000 wildebeest [6] however the population has since recovered to about 1.5 million wildebeest. While preypredator interactions involving diffusion have been applied to investigate the spatial spread of diseases $[7,8]$ the Lotka-Volterra prey predator models with diffusion have been developed to study migration of different species such as fish schools and insect swarms [6-8,9]. Surprisingly these reaction diffusion models have been less considered in modeling ungulate migration and their predators. Therefore, in this paper we present Lotka-Volterra two species prey predator reaction-diffusion model with Holling II functional response to study the dynamical behaviour of wildebeests and lions.

\section{Methods}

\section{(i) Data sources}

Data used in this study are aerial photo counts collected from Serengeti national park showing wildebeest locations in the Serengeti National Park. Data were collected April/May, 2015.In addition, we used reports and data from Tanzania Wildlife Research Institute [10].

\section{(ii) The mathematical model}

The following are the parameters were used in the model:

$r$ is the per capita intrinsic growth rates for wildebeests $u, K$ is its carrying capacity, $h$ is the capturing rates of lionsvon prey $u, a / h$ is the predator's handling time on prey, $z$ is the mortality for prey due to drought, the natural mortality rate of the predator is $c$ and the death of predator due to drought is $\mu$, the parameter $d$ measures the predator's efficiency to convert prey biomass into fertility (reproductivity), the diffusion coefficients are $D_{11}, D_{12}, D_{21}$, and $D_{22}$. Our proposed model is defined as follows:

$$
\left.\begin{array}{c}
\frac{\partial u}{d t}=\left(D_{11} \frac{\partial^{2} u}{\partial x^{2}}+D_{12} \frac{\partial^{2} u}{\partial y^{2}}\right)+r u\left(1-\frac{u}{K}\right)-\frac{h u v}{1+a u}-z u \\
\frac{\partial v}{d t}=\left(D_{21} \frac{\partial^{2} v}{\partial x^{2}}+D_{22} \frac{\partial^{2} v}{\partial y^{2}}\right)-c v+\frac{d h u v}{1+a u}-\mu v
\end{array}\right\}
$$

$u(t)>0, v(t)>0$ and all the parameters $r, K, h, z, c, d, a, \mu$ are positive.

From model equations (1), the first equation represents the prey where the first two terms represent the diffusion part and the third term is the prey logistic growth, the fourth term represents the loss of prey due to interacting with lion and the last term is the loss of prey due to drought. The second equation represents the predator where the first two terms represent the diffusion part, the third term is the natural death of predator, the fourth term is the gain of predator after a successful hunting of prey and the last term is the loss of predator due to drought.

\section{(iii) Mode analysis}

The analysis of the mathematical model involves the local and global stability analysis. This is performed to analyse the ecosystem dynamics. This is done by letting the 
Modelling Predator Prey with Diffusion for Migrating Wildebeest and Lion in the Presence of Drought

diffusive parameters $D_{11}=D_{12},=D_{21}=D_{22}=0$. The remaining prey predator model is defined below

$$
\left.\begin{array}{c}
\frac{d u}{d t}=r u\left(1-\frac{u}{K}\right)-\frac{h u v}{1+a u}-z u \\
\frac{d v}{d t}=-c v+\frac{d h u v}{1+a u}-\mu v
\end{array}\right\}
$$

\section{(iv) Boundedness of the system}

The developed mathematical model deals with living individuals, therefore, the solution of the prey-predator model developed in (2) must be positive and bounded within its region.

Lemma 1. All the solutions of the system (2) which start with $\mathbb{R}_{+}^{2}$ are uniformly bounded.

Proof: To prove the Lemma, we define a function

$$
P(t)=u(t)+\frac{1}{d} v(t)
$$

where $P(t)$ represents the total population of the prey and predator species

$$
\frac{d P}{d t}=\frac{d u}{d t}+\frac{1}{d} \frac{d v}{d t}
$$

Substitute equation (1) into equation (3) we get the following

$$
\frac{d P}{d t}=r u\left(1-\frac{u}{K}\right)-\frac{h u v}{1+a u}-z u+\frac{1}{d}\left(-c v+\frac{d h u v}{1+a u}-\mu v\right)
$$

Then all terms with interspecific competition are cancelled out, we remain with

$$
\frac{d P}{d t}=r u\left(1-\frac{u}{K}\right)-z u-\frac{1}{d}(c+\mu) v
$$

We choose the arbitrary constant to be $\Omega$ then the equation above will be written as follows

$$
\frac{d P}{d t}=r u-z u-\frac{r u^{2}}{K}-\frac{1}{d}(c+\mu) v+\Omega P(t)-\Omega P(t)
$$

Thus, $\frac{d P}{d t}+\Omega P(t) \leq r u-\frac{r u^{2}}{K}-z u-\frac{1}{d}(c+\mu) v+\Omega\left(u(t)+\frac{1}{d} v(t)\right)$

Using the concept of perfect square we obtain

$$
\frac{d P}{d t}+\Omega P(t) \leq(r-z+\Omega) u-\frac{r u^{2}}{K}-\frac{1}{d}(c+\mu-\Omega) v .
$$


Kisoma Linus, N. and Kuznetsov Dmitry

But Max $r u\left(1-\frac{u}{K}\right)$ is $\frac{K}{4 r}$, it follows

$$
\begin{aligned}
\frac{d P}{d t}+\Omega P(t) \leq & \frac{K}{4 r}(r-z-\Omega)^{2}-\frac{r}{K}\left(u^{2}-(r-z+\Omega) \frac{K}{r} u+K^{2}\left(\frac{r-z+\Omega}{4 r^{2}}\right)^{2}\right) \\
& -\frac{1}{d}(c+\mu-\Omega) v
\end{aligned}
$$

Then using techniques of completing the square it follows

$$
\frac{d P}{d t}+\Omega P(t) \leq \frac{K}{4 r}(r-z-\Omega)^{2}-\frac{r}{K}\left[u-(r-z+\Omega) \frac{K}{2 r}\right]^{2}-\frac{1}{d}(c+\mu-\Omega) v
$$

Let $\frac{K}{4 r}(r-z-\Omega)^{2}=m_{1}$, thus, $\frac{d P}{d t}+\Omega P(t) \leq m_{1}$

Solving this differential inequality using the integrating factor $I=e^{\Omega t}$ we obtained $P(t)=\frac{m_{1}}{\Omega}+C e^{-\Omega t}$.

At $t=0, P(0)=0$, then $C=-\frac{m_{1}}{\Omega}$. This gives $P(t)=\frac{m_{1}}{\Omega}\left(1-e^{-\Omega t}\right)$

As $t \rightarrow \infty, 0 \leq P(t) \leq \frac{m_{1}}{\Omega}$. Therefore, $P(t)$ is bounded. And from positivity of $u$ and $v$

$0 \leq x(t) \leq \frac{m_{1}}{\Omega}$ and $0 \leq y(t) \leq \frac{m_{1}}{\Omega}$

It follows that all the solutions of the system (2) that start in $\mathbb{R}_{+}^{2}$ are confined within the region $B$ such that $B=\left\{(u, v) \in \mathbb{R}_{+}^{2}: P=\frac{m_{1}}{\Omega}+\varepsilon\right.$, for any $\left.\varepsilon>0\right\}$.

(v) Existence of equilibrium points of the system

Taking $\frac{d u}{d t}=0$ and $\frac{d v}{d t}=0$ we get the following three equilibrium points namely

(i) $\quad E_{0}(0,0)$ is the extinction of both species, prey and predator

(ii) $\quad E_{1}\left(u^{*}, 0\right)$ is the persistence of prey and extinction of predator

(iii) $\quad E_{2}\left(u, v^{*}\right)$ is the coexistence or equilibrium point of the system

The equilibrium points $E_{0}(0,0)$ and $E_{1}\left(u^{*}, 0\right)$ are trivial and are saddle points (unstable) while $E_{2}\left(u, v^{*}\right)$ is non trivial equilibrium point (the co-existence point) with $u^{*}, v^{*}>0$

$$
\begin{gathered}
r u\left(1-\frac{u}{K}\right)-\frac{h u v}{1+a u}-z u=0 \\
-c v+\frac{d h u v}{1+a u}-\mu v=0
\end{gathered}
$$

Using MAPLE software, we obtain the following co-existence point

$$
\begin{aligned}
& E_{2}\left(u, v^{*}\right) \\
= & \left(-\frac{c+\mu}{a c+a \mu-d h},-\frac{d(K a c r-K a c z+K a \mu r-K a \mu z-K d h r+K d h z+c r+\mu r)}{K(a c+a \mu-d h)^{2}}\right)
\end{aligned}
$$

where, $a c+a \mu-d h>0$ 
Modelling Predator Prey with Diffusion for Migrating Wildebeest and Lion in the Presence of Drought

The co-existence equilibrium point exists if $a c+a \mu-d h>0$ and $r-z>0$

In the following section, we describe the local and global stability of our model. The local stability means that the equilibrium point is stable to small perturbations, such that if you push the system (put the system somewhere near the point), the system moves back to its equilibrium point whereas, the global stability means that the system will come to the equilibrium point from any possible starting point within the system.

(vi) Local stability analysis of the equilibrium points

The local asymptotic stability of each equilibrium point is studied by computing the Jacobian matrix and finding the eigenvalues evaluated at each equilibrium point. For stability of the equilibrium points, the real parts of the eigenvalues of the Jacobian matrix must be negative. From the system equations (2), the Jacobian matrix of the system is given by

$$
\begin{aligned}
& J\left(E_{i}\right)=\left(\begin{array}{ll}
\frac{\partial f_{1}}{\partial x} & \frac{\partial f_{1}}{\partial y} \\
\frac{\partial f_{2}}{\partial x} & \frac{\partial f_{2}}{\partial y}
\end{array}\right) \\
& J\left(E_{3}\right)=\left(\begin{array}{cc}
r-\frac{2 r u^{*}}{K}-\frac{h v^{*}}{\left(1+a u^{*}\right)}+\frac{h a u^{*} v^{*}}{\left(1+a u^{*}\right)^{2}}-z & \frac{-h u^{*}}{\left(1+a u^{*}\right)} \\
\frac{d h v^{*}}{\left(1+a u^{*}\right)}-\frac{d h a u^{*} v^{*}}{\left(1+a u^{*}\right)^{2}} & -c+\frac{d h u^{*}}{1+a u^{*}}-\mu
\end{array}\right) \\
& \begin{aligned}
& E_{2}\left(u^{*}, v^{*}\right) \\
= & \left(-\frac{c+\mu}{a c+a \mu-d h},-\frac{d(K a c r-K a c z+K a \mu r-K a \mu z-K d h r+K d h z+c r+\mu r}{(a c+a \mu-d h)^{2}}\right)
\end{aligned} \\
& J\left(E_{2}\right)=\left(\begin{array}{ll}
E_{11} & E_{12} \\
E_{21} & E_{22}
\end{array}\right)
\end{aligned}
$$

Using MAPLE software we get the following:

$$
\begin{gathered}
E_{11}=\frac{1}{4} \frac{1}{K\left((c+\mu) a-\frac{1}{2} d h\right)^{2}((c+\mu) a-d h)}\left(4 K(c+\mu)^{3}(r-z) a^{3}\right. \\
-9\left(d h(r-z) K+\frac{8}{9} r(c+\mu)\right)(c+\mu)^{2} a^{2} \\
+7 d h(c+\mu)(d h(r-z) K+r(c+\mu)) a \\
\left.-2\left(d h(r-z) K+\frac{1}{2} r(c+\mu)\right) d^{2} h^{2}\right) \\
E_{12}=\frac{(c+\mu) h}{(2 c+2 \mu) a-d h}
\end{gathered}
$$


Kisoma Linus, N. and Kuznetsov Dmitry

$$
\begin{gathered}
E_{21}=\frac{1}{4} \frac{((r-z)((c+\mu) a-d h) K+r(c+\mu)) d^{2} h}{K\left((c+\mu) a-\frac{1}{2} d h\right)^{2}} \\
E_{22}=\frac{2(c+\mu)((c+\mu) a-d h)}{(2 c+2 \mu) a-d h}
\end{gathered}
$$

The stability of the $J\left(E_{2}\right)$ is stated using the the characteristic of polynomial equation techniques using trace and determinant techniques proposition as follows

Preposition 1. Suppose the Jacobian matrix evaluated at the coexistence equilibrium point has a characteristic polynomial equation of the following form:

$$
\lambda^{2}-\operatorname{trace}\left(J\left(E_{2}\right)\right) \lambda+\operatorname{Determinant}\left(J\left(E_{2}\right)\right)=0
$$

such that

$\operatorname{trace}\left(J\left(E_{2}\right)\right)=E_{11}+E_{22}$ andDeterminant $\left(J\left(E_{2}\right)\right)=E_{11} E_{22}-E_{12} E_{21}$

The co-existence equilibrium point is locally asymptotically stable or stable spiral if trace $\left(J\left(E_{2}\right)\right)<0$ and Determinant $\left(J\left(E_{2}\right)\right)>0$. Also the interior equlibrium point is center (neutral stable) if trace $\left(J\left(E_{2}\right)\right)=0$ and Determinant $\left(J\left(E_{2}\right)\right)>0$.

\section{(vii) Global Stability of equilibrium points}

Points $E_{1}$ and $E_{2}$ are shown by Linearizing the system of equations (2) and defining appropriate Lyapounov function to separately described each equilibrium point. The Linearization process is done using Jacobian technique such that

$$
\frac{d U_{i}}{d t}=J\left(E_{i}\right) U_{i}
$$

where $J\left(E_{i}\right)$ is the Jacobian Matrix and $U_{i}$ is the small perturbation on $u_{i}$. Therefore system (1) reduces to the following linear system;

$$
\left.\begin{array}{c}
\frac{d U}{d t}=\left[r-\frac{2 r u^{*}}{K}-\frac{h v^{*}}{\left(1+a u^{*}\right)}+\frac{h a u^{*} v^{*}}{\left(1+a u^{*}\right)^{2}}-z\right] U-\left[\frac{h u^{*}}{\left(1+a u^{*}\right)}\right] V \\
\frac{d V}{d t}=\left[\frac{d h v^{*}}{\left(1+a u^{*}\right)}-\frac{d h a u^{*} v^{*}}{\left(1+a u^{*}\right)^{2}}\right] U+\left[-c+\frac{d h u^{*}}{1+a u^{*}}-\mu\right] V
\end{array}\right\}
$$

We choose the following Lyapunov function

$$
Q(U, V)=\frac{U^{2}}{2}+\frac{V^{2}}{2}
$$

The function $Q(U, V)$ is positive definite since $Q(U, V) \geq 0, \forall(U, V)$.

$$
\frac{\partial Q}{\partial U}=U, \quad \frac{\partial Q}{\partial V}=V
$$

The time derivative of $Q(U, V)$ is given by 
Modelling Predator Prey with Diffusion for Migrating Wildebeest and Lion in the Presence of Drought

$$
\frac{d Q}{d t}=\frac{\partial Q}{\partial U} \frac{d U}{d t}+\frac{\partial Q}{\partial V} \frac{d V}{d t}
$$

Substituting equation (4) into equation (5) we get the following relation

$$
\begin{aligned}
& \frac{d Q}{d t}=U\left[\left(r-\frac{2 r u^{*}}{K}-\frac{h v^{*}}{\left(1+a x^{*}\right)}+\frac{h a u^{*} v^{*}}{\left(1+a u^{*}\right)^{2}}-z\right) U-\left(\frac{h u^{*}}{\left(1+a u^{*}\right)}\right) V\right]+V\left[\left(\frac{d h v^{*}}{\left(1+a u^{*}\right)}-\right.\right. \\
& \left.\left.\frac{d h a u^{*} v^{*}}{\left(1+a u^{*}\right)^{2}}\right) U+\left(-c+\frac{d h u^{*}}{1+a u^{*}}-\mu\right) V\right]
\end{aligned}
$$

For a fixed point $E_{1}\left(u^{*}, 0\right)$

We substitute $E_{1}\left(u^{*}, 0\right)=E_{1}\left(\frac{K(r-z)}{r}, 0\right)$ to equation (6) we get

$$
\frac{d Q}{d t}=U\left[\left(r-\frac{2 r u^{*}}{K}-z\right) U-\left(\frac{h u^{*}}{\left(1+a u^{*}\right)}\right) V\right]+V\left[\left(-c+\frac{d h u^{*}}{1+a u}-\mu\right) V\right]
$$

This simplifies to

$$
\frac{d Q}{d t}=U^{2}(-r+z)-\left(\frac{h K(r-z)}{r+a K(r-z)}\right) U V+\left(-c+\frac{d h K(r-z)}{r+a K(r-z)}-\mu\right) V^{2}
$$

Therefore from the equation $(7)$ the equilibrium point $E_{1}\left(u^{*}, 0\right)$ is globally asymptotically stable if $(r-z)>0$

Hence, in the absence of the predator, point $E_{1}\left(u^{*}, 0\right)$ is globally stable if the intrinsic growth rate of the prey population is greater than the natural mortality rate.

For steady state $E_{2}\left(u^{*}, v^{*}\right)$

We substituted

$$
\begin{aligned}
& E_{2}\left(u^{*}, v^{*}\right) \\
= & \left(-\frac{c+\mu}{a c+a \mu-d h},-\frac{d(K a c r-K a c z+K a \mu r-K a \mu z-K d h r+K d h z+c r+\mu r}{(a c+a \mu-d h)^{2}}\right)
\end{aligned}
$$

into equation (6) we obtained an equation of the following form

$$
\frac{d Q}{d t}=E_{11} U^{2}+\left(E_{12}+E_{21}\right) U V
$$

Therefore the point $E_{2}\left(u^{*}, v^{*}\right)$ is globally stable if the condition below holds

$$
\frac{d Q}{d t}=\left(E_{11} U^{2}+\left(E_{12}+E_{21}\right) U V\right)<0
$$

(viii) Model analysis with diffusion

From the system of equations (1) 
Kisoma Linus, N. and Kuznetsov Dmitry

$$
\left.\begin{array}{c}
\frac{\partial u}{d t}=\left(D_{11} \frac{\partial^{2} u}{\partial x^{2}}+D_{12} \frac{\partial^{2} u}{\partial y^{2}}\right)+r u\left(1-\frac{u}{K}\right)-\frac{h u v}{1+a u}-z u \\
\frac{\partial v}{d t}=\left(D_{21} \frac{\partial^{2} v}{\partial x^{2}}+D_{22} \frac{\partial^{2} v}{\partial y^{2}}\right)-c v+\frac{d h u v}{1+a u}-\mu v
\end{array}\right\}
$$

\section{(ix) Numerical solution}

In this section we use the implicit Euler method to approximate solutions to the model equations (1) subject to the boundary conditions $u(x, 0, t)=u\left(L_{x}, 0, t\right)=0, u(0, y, t)=$ $u\left(0, L_{y}, t\right)=0$ and initial conditions $u(x, y, 0)=u_{0}$ and $v(x, y, 0)=v_{0}$ Such that $0<x<L_{x}, 0<y<L_{y}$ and $0<t<T$. We chose $L_{x}=1, L_{y}=4$ and $T=1000$. The time derivative is discretized by using forward difference rule and while the spatial derivatives are discretized using the central difference approximation as shown below

$$
\begin{gathered}
\frac{u_{i, j}^{n+1}-u_{i, j}^{n}}{n}=D_{11}\left(\frac{u_{i+1, j}^{n}-2 u_{i, j}^{n}+u_{i-1, j}^{n}}{h^{2}}\right)+D_{12}\left(\frac{u_{i, j+1}^{n}-2 u_{i, j}^{n}+u_{i, j-1}^{n}}{h^{2}}\right) \\
+r u_{i, j}^{n}\left(1-\frac{u_{i, j}^{n}}{K}\right)-\frac{h u_{i, j}^{n} v_{i, j}^{n}}{1+a u_{i, j}^{n}}-z u_{i, j}^{n} \\
\frac{v_{i, j}^{n+1}-v_{i, j}^{n}}{k}=D_{21}\left(\frac{v_{i+1, j}^{n}-2 v_{i, j}^{n}+v_{i-1, j}^{n}}{h^{2}}\right)+D_{22}\left(\frac{v_{i, j+1}^{n}-2 v_{i, j}^{n}+v_{i, j-1}^{n}}{h^{2}}\right)-c v_{i, j}^{n}+\frac{d h u_{i, j}^{n} v_{i, j}^{n}}{1+a u_{i, j}^{n}}-\mu v_{i, j}^{n}(9)
\end{gathered}
$$

Parameters and their sources are shown in the following table.

\begin{tabular}{|l|c|l|l|}
\hline Parameter Description & Symbol & Value & Source \\
\hline Per capita intrinsic growth rate for prey & $r$ & 1 & Mduma, 1996 \\
\hline Natural mortality rate of predator & $c$ & 1.2 & Schaller, 1972 \\
\hline Mortality rate of prey due to drought & $z$ & 0.15 & Sinclair et al. 2008 \\
\hline Capturing rates of the predator & $h$ & 0.674 & Fryxell et al. 2007 \\
\hline Prey biomass handled per unit time & $a$ & 0.03 & Assumed \\
\hline $\begin{array}{l}\text { Efficiency to convert prey biomass into } \\
\text { fertility }\end{array}$ & $d$ & 0.18 & Assumed \\
\hline Mortality rate of predator due to drought & $\mu$ & 0.01 & Sinclair et al. 2008 \\
\hline
\end{tabular}

Table 1: Parameters of the prey predator model with their sources

\section{Results of the prey predator model}

We simulated the current estimates of wildebeest population $\left(u_{0}=1.3\right.$ million $)$ and lion $\left(v_{0}=3,000\right)$ and used parameters in table 1 . In the first case, the simulations for prey-pre dator are done in absence of drought. We observed thatPhase diagram for model (2) show $\mathrm{s}$ a stable spiral (sink) between lion and wildebeest. Hence in absence of any other factor in Serengeti ecosystem, the dynamical system is stable spiral around the point $(10.18,0.8$ 8 ) i.e., 1.018 million wildebeests and 88,000 lions (see fig 1). 
Modelling Predator Prey with Diffusion for Migrating Wildebeest and Lion in the Presence of Drought
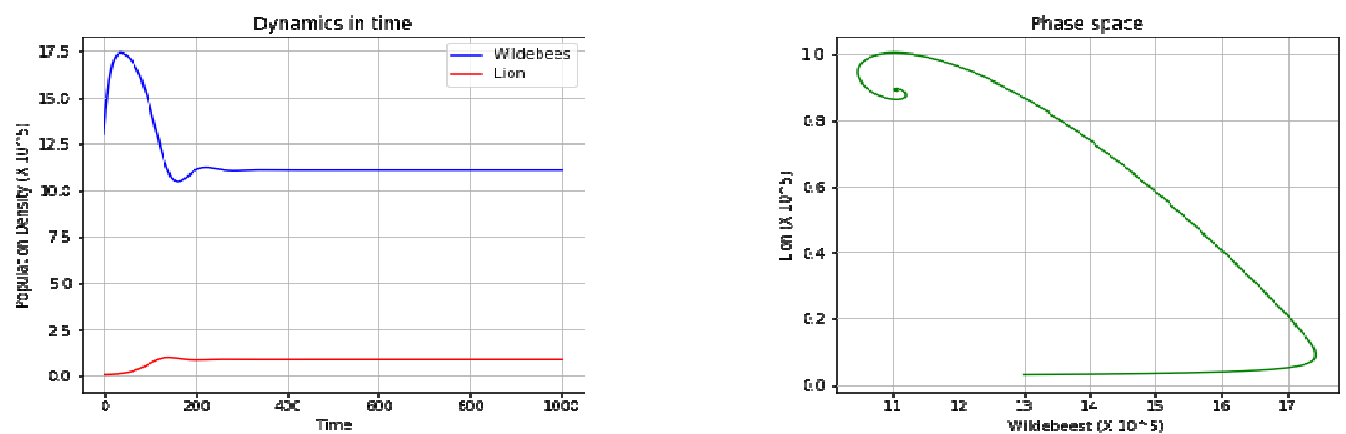

Figure 1: Population Dynamics of Lion and wildebeest $(z=0, \mu=0)$

The major limiting factor for wildebeest growth is availability of enough forage. We vari ed the parameter of drought for both prey and predator. We observed that for moderate dr ought seasons, the abundance of both prey and predator grows slowlytoabout $(8.5,0.66) \mathrm{i}$. e., 850,000 wildebeest and 66,000 lion (fig. 2).
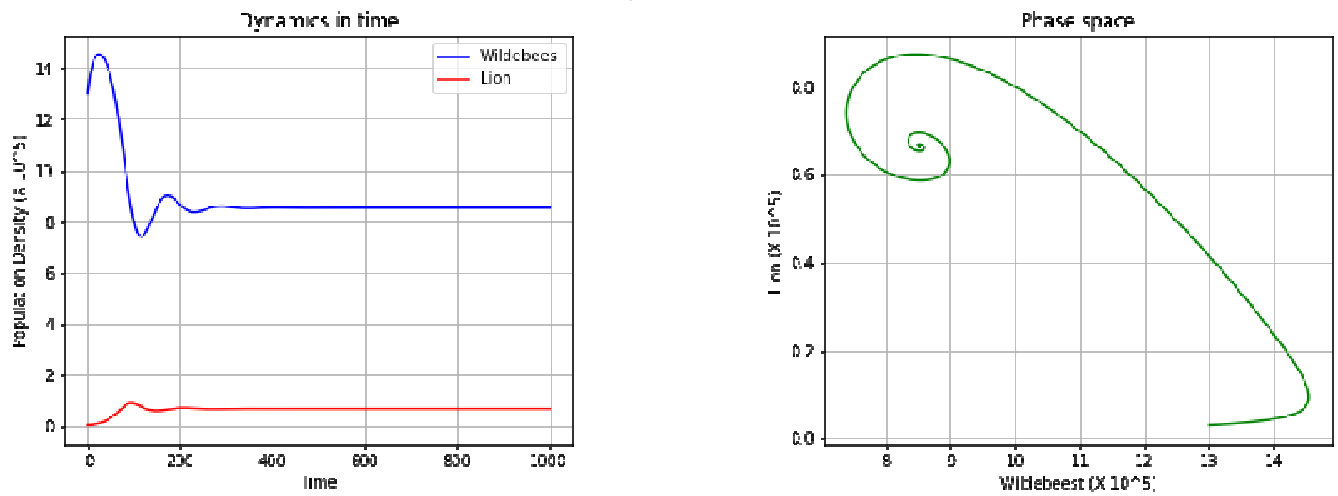

Figure 2: Population Dynamics of lion and wildebeest during moderate drought seasons $z=0.15$ and $\mu=0.01$.

When wildebeest population was declining in Kruger national park in South Africa, the average birthrate was 0.405 and the hunting increases from 0.674 to 0.81 (Fay \& Greef, 1999a.). We used this birth rate and hunting efficiency for the declining wildebeest population and we observed a decline wildebeest and lion population when drought persists (see fig 3). 
Kisoma Linus, N. and Kuznetsov Dmitry
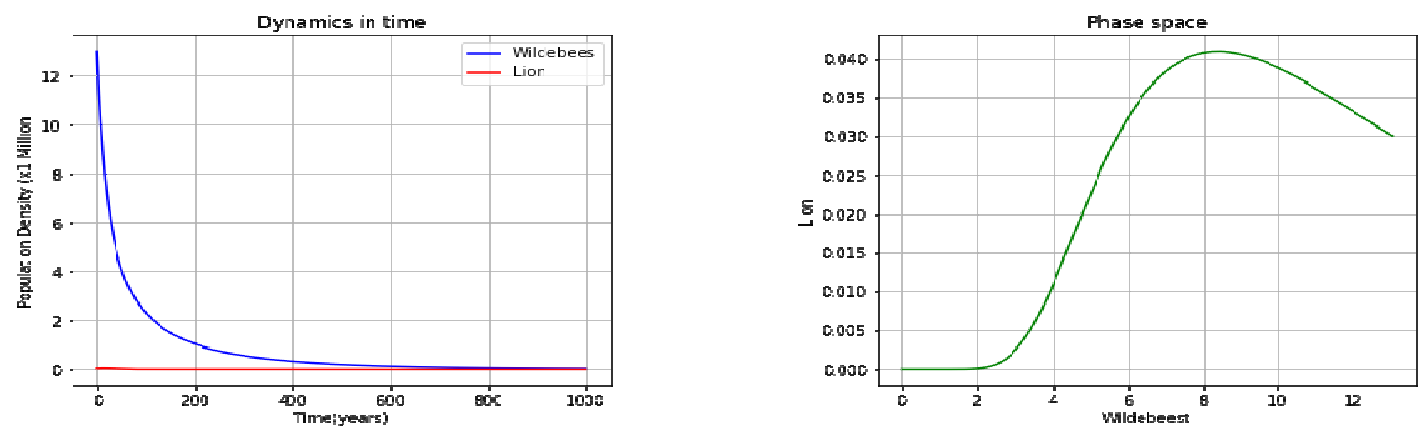

Figure 3: Population dynamics of Lion and wildebeest in presence of severe drought $z=0.45, \mu=0.02$ and $r=0.405, h=0.81$

Furthermore, diffusion of wildebeest and lion yielded regular peaks and troughs of prey and predator. This behaviour is shown in figure 4 and 5 for prey and predator populations.

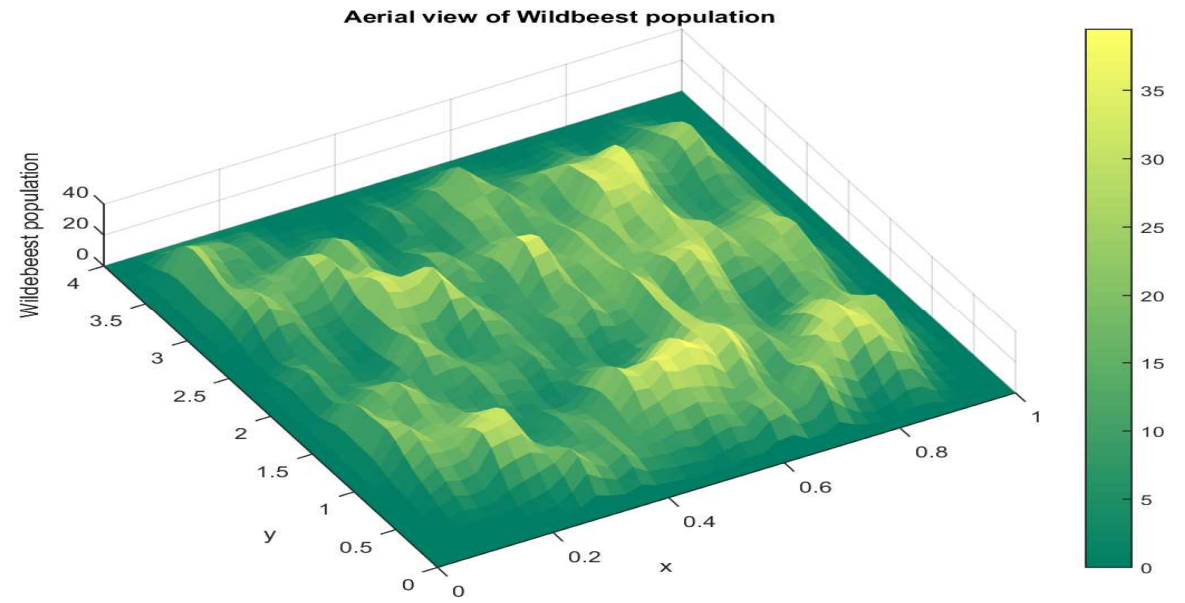

Figure 4: Aerial view of wildebeest population patterns due to diffusion 
Modelling Predator Prey with Diffusion for Migrating Wildebeest and Lion in the Presence of Drought

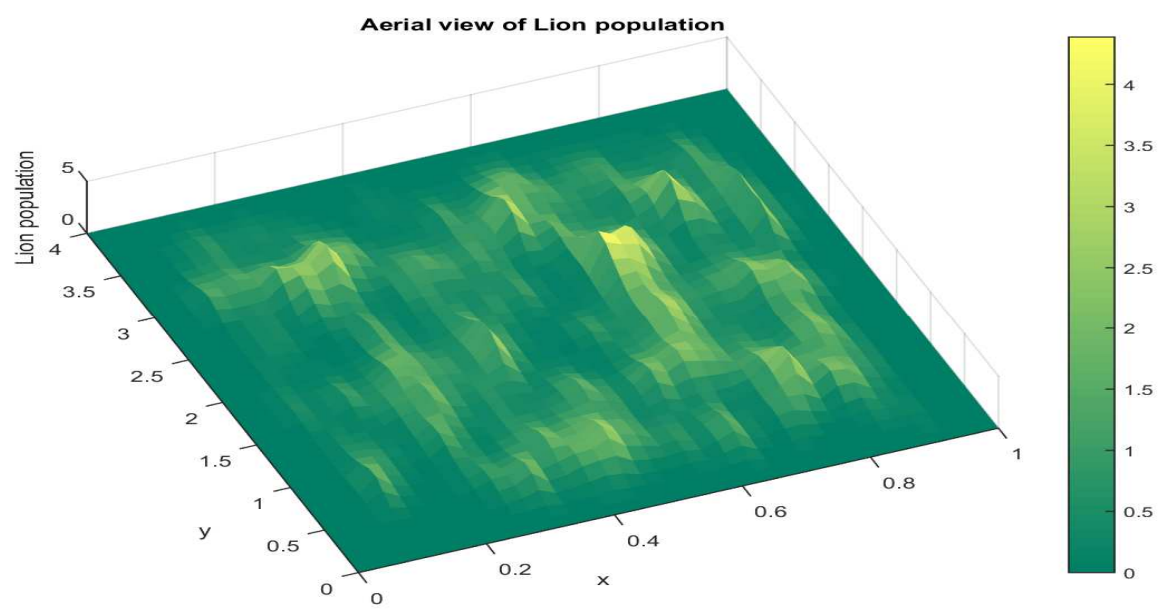

Figure 5: Aerial view of lion population patterns due to diffusion

Figures 4 and 5 are aerial view illustrating the type of patterns that can be formed when a predator-prey model is subjected to diffusion.

However, if the intensity of drought increases we observed decreased population of wildebeest and lion as shown in figures 6 and 7.

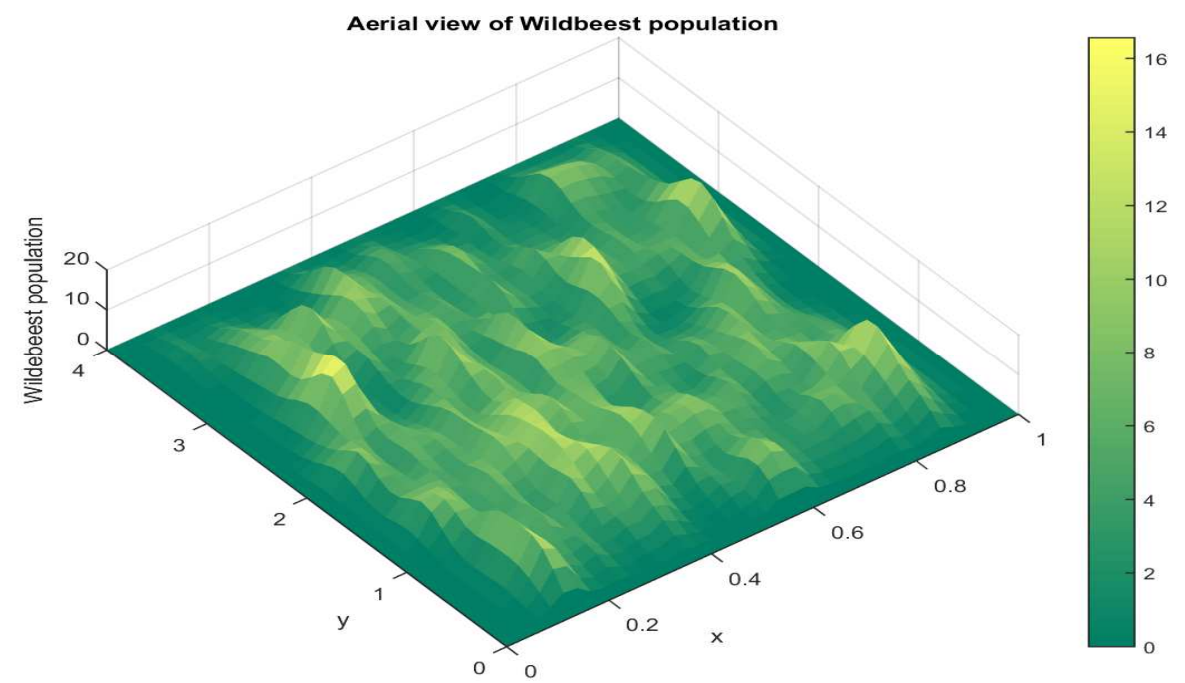

Figure 6: Aerial view of lion population patterns due to diffusion and drought 
Kisoma Linus, N. and Kuznetsov Dmitry

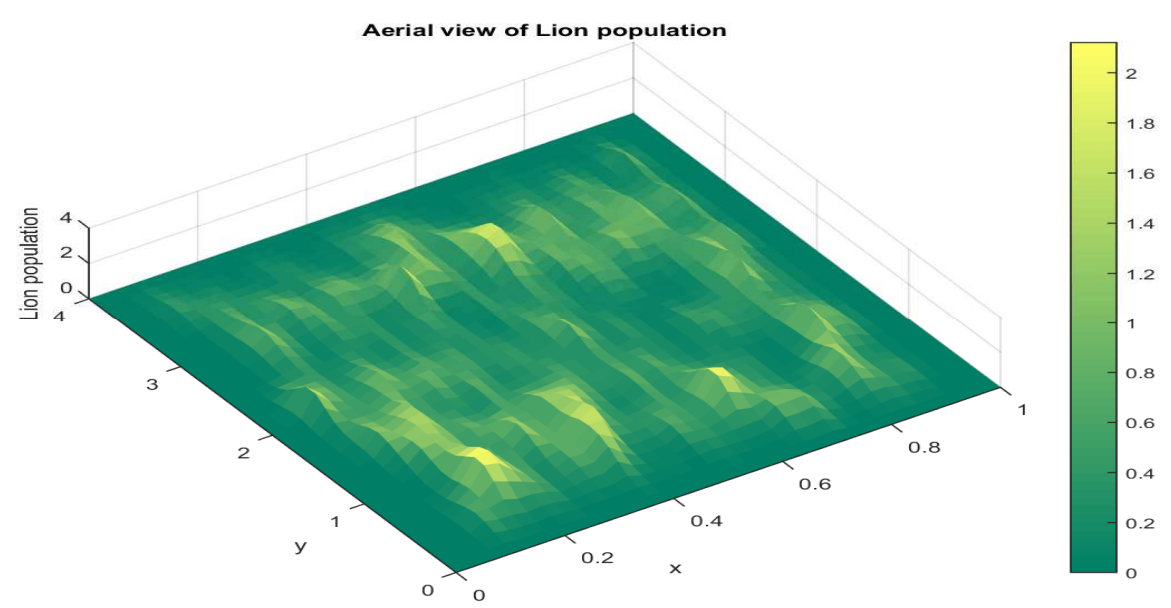

Figure 7: Aerial view of lion population patterns due to diffusion and drought.

We observe from the color bar a decreased population.

\section{Conclusion}

The dynamical system of migrating wildebeest followed by lion in the Serengeti ecosystem shows a stable state. However, in presence of drought the population of both species decreases and hence we observed unstable state as drought persisted. Since forage is the main driver of wildebeest migration, wildebeest evolve migratory pathways containing information on the availability ofgood forage and free from predators. Therefore, diffusion of wildebeest followed by predators from place to place within Serengeti ecosystem is a result of search for better forage availability. Further, the Serengeti lion usually hide to areas with high prey catchability like woody vegetation and near water sources to catch prey. This relation leads to diffusion processes. The developed PDEs have successfully described the dynamical behaviour of migrating prey and predator.

\section{REFERENCES}

1. T.Damas Sagamiko, N. Shaban, C.L Nahonyo and O.D.Makinde, Optimal control of a threatened wildebeest-lion prey-predator system in the serengeti ecosystem, Appl Comput Math, 4(4) (2015) 296-312.

Available from: http://www.sciencepublishinggroup.com/j/acm

2. T.H.Fay and J.C.Greeff, Lion, wildebeest and zebra: A predator-prey model, Ecol Modelling, 196(1-2) (2006) 237-244.

3. A.Rusliza and B.Harun, Stability analysis of mutualism population model with time delay, Int J Math Comput Sci., 6(2) (2012) 34-45.

4. D.Ikanda and C.Packer, Ritual vs. retaliatory killing of African lions in the Ngorongoro Conservation Area, Tanzania, Endanger Species Res., 6(1) (2008) 67-74.

5. R.M.Holdo, J.M.Fryxell, A.R.E.Sinclair, A.Dobson and R.D.Holt, Predicted impact of barriers to migration on the Serengeti wildebeest population, PLoS One, 6(1) (2011) 56-78.

6. S.A.R.Mduma, A.R.E.Sinclair and R.Hilborn, Food regulates the Serengeti Wilde - 
Modelling Predator Prey with Diffusion for Migrating Wildebeest and Lion in the Presence of Drought

beest: A 40 year Record, J Anim Ecol., 68(6) (1999) 1101-1122.

7. E.E.Holmes, M.A.Lewis, J.E.Banks, R.R.Veit and N.Jan, Partial differential equations in ecology: spatial interactions and population dynamics, Ecology, 75(1) (1994) 17-29.

8. A.Okubo and S.A.Levin, Diffusion and ecological problems modern perspectives. Springer. 2001.

9. A.Okubo. Dynamical aspects of animal grouping: Swarms, schools, flocks, and herds, Adv Biophys, 22(C) (1986) 1-94.

10. T.M.Edeus, T.M.Honori, K.B.Samwel and K.Hamza, Tanzania Wildlife Research Institute (TAWIRI), 2010 Aerial Census in the Serengeti Ecosystem. 2010.

11. T.H.Fay and J.C.Greef, Lion and Wildebeest: a prey predator model, Math Comput Educ., 25(2) (2015) 33-45. 